\title{
Congenital neuroblastoma
}

\author{
A. R. EVANS \\ From the Group Laboratory, the Royal Infirmary, Wigan
}

SYNOPSIS The clinical histories and post-mortem findings in five cases of neuroblastoma are described, and an account given of the microscopic characteristics of the tumours. In four of the cases the tumour was present at birth and was probably so in the fifth case. In only one case was the presence of the malignant tumour a significant factor in causing death. The differential diagnostis of such tumours is discussed.

The accumulated evidence of many recorded cases suggests that neuroblastoma, becoming manifest in the early months or weeks of life, and congenital tumour, would be included in such group, and has an appreciably better prognosis than has this same tumour when it becomes manife\& in later childhood. The literature is briefly reviewed to illustrate this aspect of prognosis and possiblet reasons for it are indicated.

With the possible exception of retinoblastoma, congenital malignant tumours are not common.

Wells (1940), in an extensive and critical review of the available literature to determine the occurrence and significance of malignant tumours present at or before birth, accepted 66 reported cases as established beyond doubt. Of these tumours 33 were sarcomata and 23 neuroblastoma. The neuroblastomata included four cases encountered by the reviewer in a series of 3,000 necropsies on newborn and stillborn infants at the University of Chicago Hospitals. In summary Wells emphasized that while half of the established congenital tumours were sarcomata, there were very nearly as many cases of neuroblastoma and that a vastly larger proportion of all recorded neuroblastoma (about 275 cases had been reported in the literature up to 1938) was present at birth as compared with the relatively small proportion of congenital sarcomata.

Instances of congenital neuroblastoma recorded since this review by Wells include the cases described by Hepler (1943), Larimer (1949), Beck and Howard (1951), and Haber and Bennington (1963).

Five further cases, in four of which the tumour was certainly present at birth, and was probably so in the fifth, are described in this report. Three of them formed part of a total of 59 cases of neuroblastoma registered at the Children's Tumour Registry of the University of Manchester in the nine-year period September 1953 to September 1962. I observed the remaining two cases in a series of 28 necropsies on stillborn and newborn infants at the Billinge Hospital, Orrell, during the year 1963.

Received for publication 8 April 1964.
CASE 1

The mother was a primigravida aged 25 years. Pregnane was complicated by mild toxaemia but terminated in normal labour with the birth of a male child weighing $7 \mathrm{lb} .3 \mathrm{oz}$. The child was 'slow to gasp' and, despie clearance of airways of mucus and oxygen administration respiration remained impaired. Clinical examination revealed a tense swelling in the upper abdomen. The had a firm lower edge palpable just below the level of the umbilicus. Peripheral blood examination showed haemoglobin level of $13.3 \mathrm{~g} . / 100 \mathrm{ml}$.; leucocytes 18,100 , c.mm. (neutrophils $60 \%$, lymphocytes $37 \%$, monocytes $2 \%$, eosinophils $1 \%$ ), The film showed no abnormal white cells, and a degree of polychromasia with occasion late normoblasts which was considered to be within the normal limits for a child of this age. The blood urea leve was $36 \mathrm{mg} . / 100 \mathrm{ml}$. An intravenous pyelogram outlinẹg an apparently normal renal tract. Respiratory distre became progressive, despite continuous oxygen admin stration and other restorative measures, and the generg condition deteriorated rapidly. Death occurred 34 hours after birth.

\section{NECROPSY}

There was no evidence of birth trauma. The face and finger nail beds were deeply cyanosed.

The lungs were congested and showed widespre patchy atalectatic areas.

The left adrenal was occupied by a soft rounde्ल lobulated vascular medullary tumour $3 \times 2 \times$ $1.5 \mathrm{~cm}$. The cut surface was dark red, mottled with lighter pink and yellow areas and showed incomplete rim of recognizable cortex. The tumoor was well demarcated, compressing but not infiltra ing the upper pole of the left kidney (Fig. 1). 


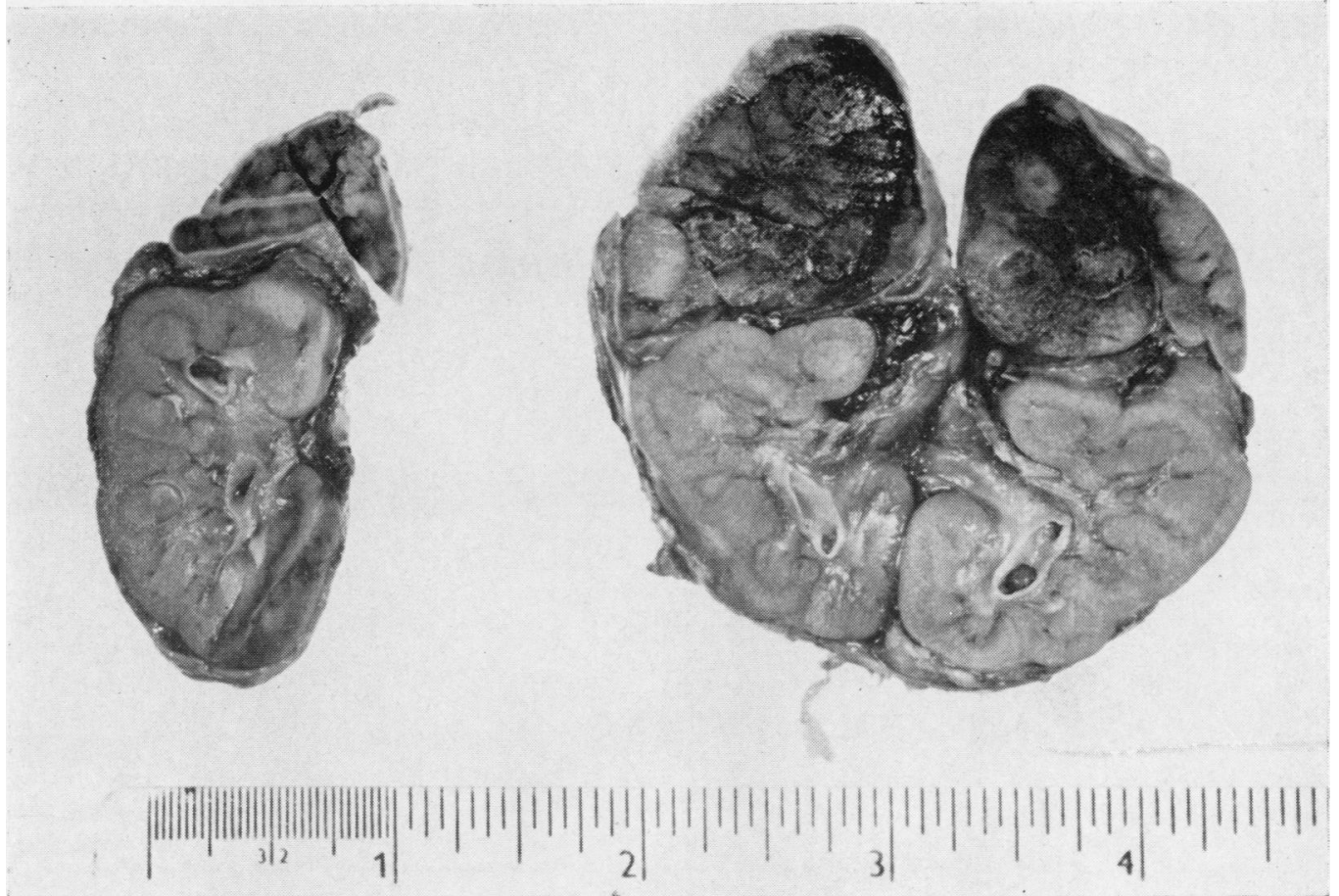

FIG. 1. Left adrenal neuroblastoma compressing upper pole of kidney. Small pale tumour nodules are seen in the medulla of the right adrenal (case 1).

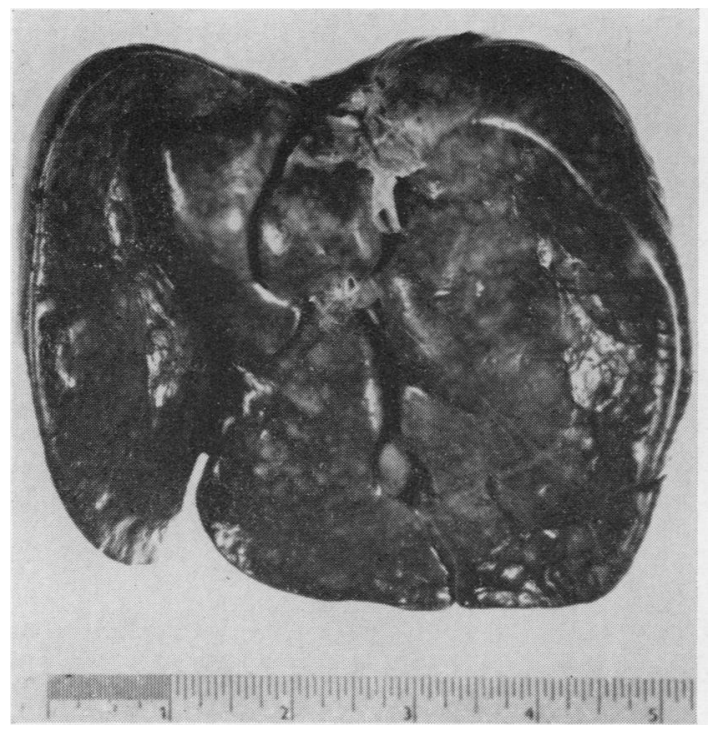

FIG. 2

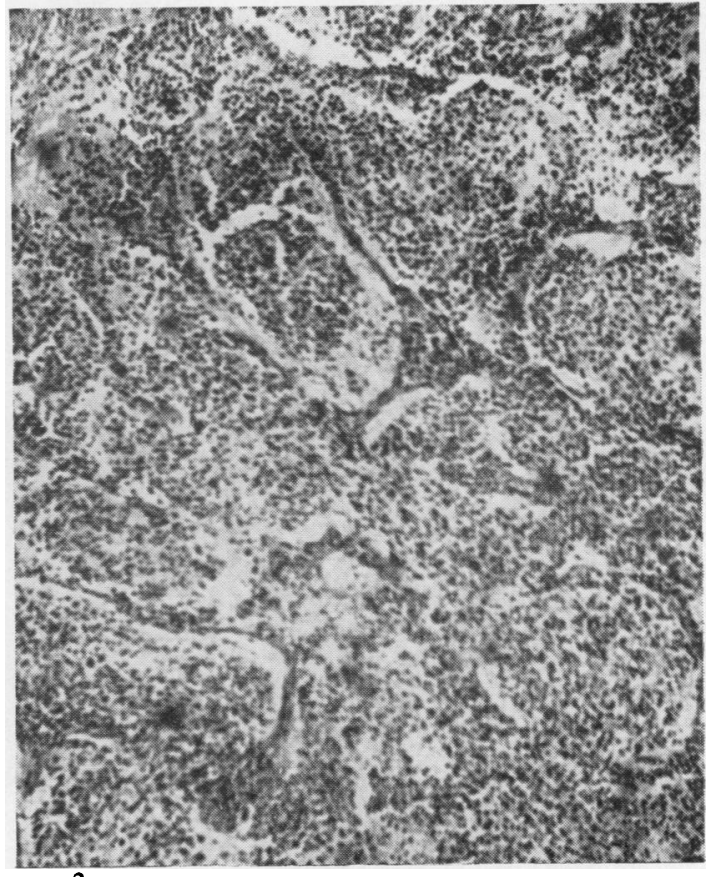

FIG. 3

FIG. 2. Undersurface of liver in case 1 showing nodules of tumour seen as pale areas through the capsule.

FIG. 3. Left adrenal tumour in case 1 showing the characteristic lobular pattern. Haematoxylin and eosin $\times 80$. 


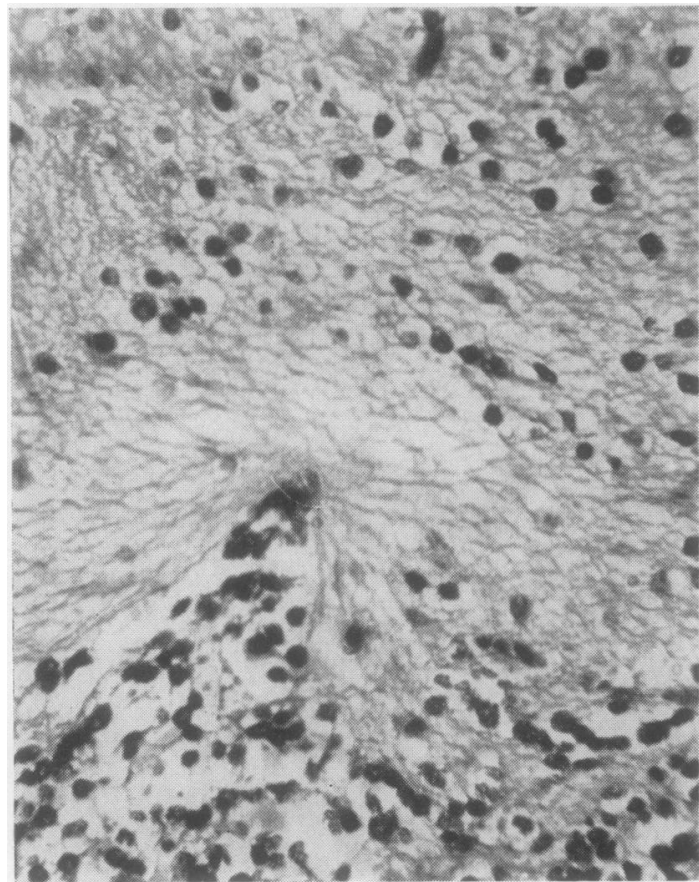

FIG. 4

FIG. 4. Neuroblastoma (case 1) showing abundant neurofibrillary

FIG. 5. Neuroblastoma (case 1) showing two rosettes in a haemorrhagic area of the tumour. Haematoxylin a eosin $\times 320$.

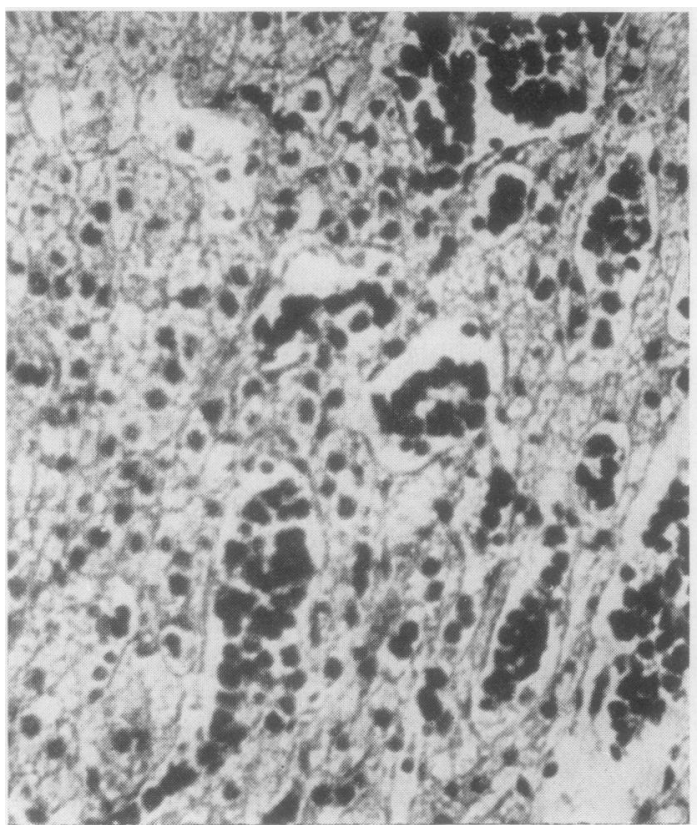

FIG. 6. Infiltration of adrenal cortex by neuroblastoma (case 1). Haematoxylin and eosin $\times 320$.

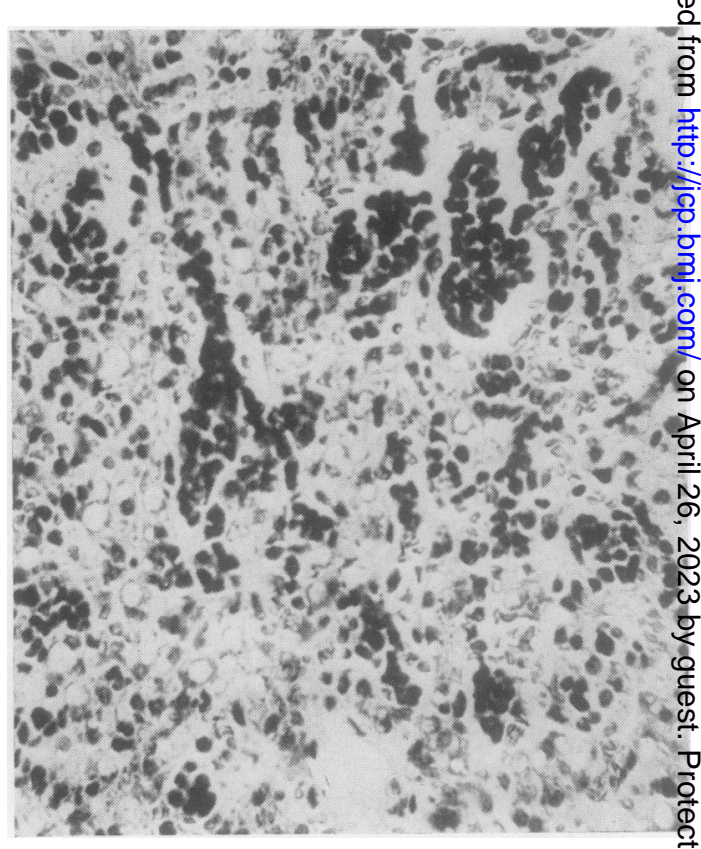

FIG. 7. Liver (case 1) showing neuroblastomatous infilt tion. Haematoxylin and eosin $\times 320$.
穴

을 
The right adrenal appeared normal in size and shape but the cut surface showed a few small grey tumour nodules in the medulla, each measuring 1 to $2 \mathrm{~mm}$. in diameter.

The liver was large, weighing $600 \mathrm{~g}$. The edges were smooth and rounded and the whole of the surface was mottled by numerous low nodules of greyish yellow tumour tissue varying in diameter from 1 to $5 \mathrm{~mm}$. The cut surface showed a similar pattern of tumour infiltration (Fig. 2).

A single para-aortic lymph node was enlarged by tumour. The remaining organs showed no abnormality apart from varying degrees of congestion.

\section{HISTOLOGY}

LEFT ADRENAL Sections showed a neuroblastoma. The tumour cells were small with darkly staining nuclei showing rather dense chromatin and obscure intranuclear detail. Nuclear diameter varied from about $8 \mu$ to $14 \mu$. In the smallest cells the nuclei were deeply and evenly staining with only an inconspicuous rim of scanty cytoplasm. Such lymphocyte-like cells have been called sympathogonia from their resemblance to those cells of neuroectodermal origin which are the formative cells of the sympathetic nervous system (Blacklock, 1934). Most of the tumour cells were somewhat larger having a rather more open coarsely vesicular, nuclear chromatin pattern and sometimes showing more plentiful cytoplasm. The larger cells have been described as sympathoblasts, again from their resemblance to those cells in developing sympathetic nervous tissue which are derived from sympathogonia and represent a first step in differentiation towards mature ganglion cells.

Architecturally the tumour presented a lobulated pattern (Fig. 3) with sheets of tumour cells divided by a vascular supporting stroma into acinar areas of varying diameter. The stroma varied in density from broad bands of mature fibrous tissue in some areas to delicate strands of capillaries with little connective tissue in others. Within the lobules the tumour cells showed a characteristic 'loosely packed' pattern with an abundance of eosinophilic fibrillary material arranged as a loose meshwork or as parallel bundles of fibrils lying between the cells and cell groups (Fig. 4). Occasional individual fibrils were seen arising as extensions from the cytoplasm of tumour cells and some small groups of cells showed ball-like clumping and sometimes a circular arrangement around a central core of fibrillary or granular material (Fig. 5). Such rosettes, often described as a characteristic feature of neuroblastoma, were ill formed and scarce.

Numerous areas of haemorrhage were seen throughout the tumour and the incomplete rim of rather stretched cortex was extensively infiltrated by tumour (Fig. 6).

RIGHT ADRENAL The medulla contained areas of neuroblastoma and cortical infiltration was evident.

LIVER Normal hepatic architecture was obliterated by extensive tumour infiltration (Fig. 7). Short cords of liver cells with occasional haemopoietic foci were widely separated from one another by strands and sheets of tumour cells, and in some areas tumour cells were seen lying within portal vessels. The tumour cells were similar to those seen in the primary growth but fibrillary substances was not plentiful and of course the lobular pattern of the primary tumour was not apparent.

\section{CASE 2}

The mother was 40 years of age and had had three previous pregnancies, all normal, and all three of the children were alive and well. The current pregnancy was normal but terminated with premature rupture of membranes followed by breech delivery of a male child ( $5 \mathrm{lb} .10 \mathrm{oz}$.) some 55 hours later. Satisfactory normal respiration was never established and the child died within 30 minutes of birth.

\section{NECROPSY}

There was no evidence of birth trauma. The face and nail beds were deeply cyanosed.

The lungs showed extensive atalectasis and were congested with a few small areas of pulmonary haemorrhage.

The left adrenal contained a soft, rounded, demarcated medullary tumour measuring $3 \times 3 \times$

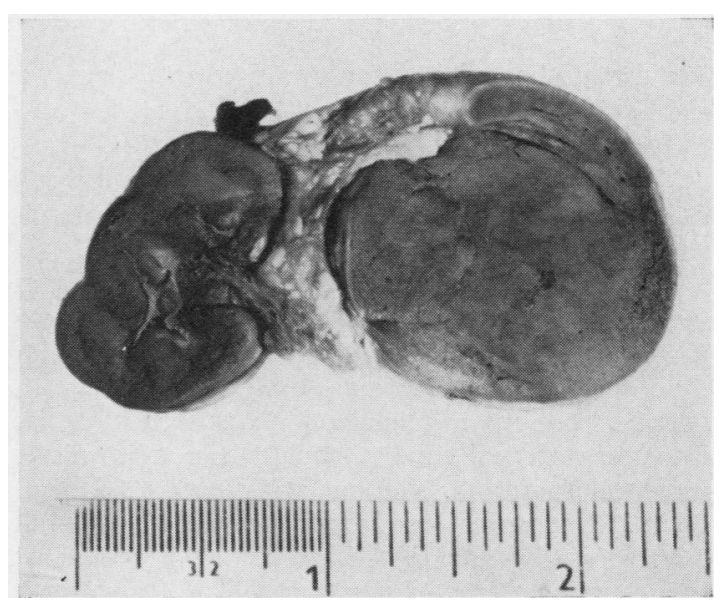

FIG. 8. Left adrenal tumour (case 2). 
$2 \mathrm{~cm}$. The cut surface was uniformly deep red and there was a partial rim of thin cortex at its periphery (Fig. 8).

The right adrenal was macroscopically normal.

There was no evidence of tumour metastases and apart from venous congestion the remaining organs showed no significant abnormality.

\section{HISTOLOGY}

LEFT ADRENAL The tumour was a neuroblastoma presenting a similar histological picture to that described in case 1 with a lobular architecture and abundant fibrillary material. The tumour cells showed similar small variations in size, nuclear pattern, and amount of cytoplasm, indicative of partial differentiation. Rosettes were not plentiful and for the most part incompletely elaborated. There were numerous small areas of haemorrhage but no evidence of necrosis or calcification. The adrenal cortex showed infiltration by tumour cells.

RIGHT ADRENAL Although macroscopically normal, the medulla contained neuroblastoma with several areas of early cortical infiltration.

CASE 3

The patient was a male child born at home in October 1954. Pregnancy had been normal and the birth weight was $5 \mathrm{lb} .4 \mathrm{oz}$. Delivery was reported as normal except that the umbilical cord was wrapped three times round the infant's neck. The child remained well for 24 hours then became cyanosed and limp and was admitted to Hope Hospital, Manchester. The general condition deteriorated and death occurred about 38 hours after birth.

\section{NECROPSY}

The body showed general cyanosis and there was right-sided talipes.

The lungs showed slight collapse.

The brain was congested, with general subarachnoid oozing and considerable subtentorial haemorrhage.

A firm but soft white slightly lobulated paravertebral tumour measuring $1.5 \times 1 \times 0.5 \mathrm{~cm}$. was present behind the right kidney and partly covered by the psoas muscle. It was closely attached to the vertebral column and on section was seen to project into the vertebral canal through three of the intervertebral foramina. The cut surface was uniformly white. A second minute white tumour nodule, $2.5 \mathrm{~mm}$. in diameter, was found in the paravertebral tissues above the main tumour.

The remaining organs showed no abnormality.

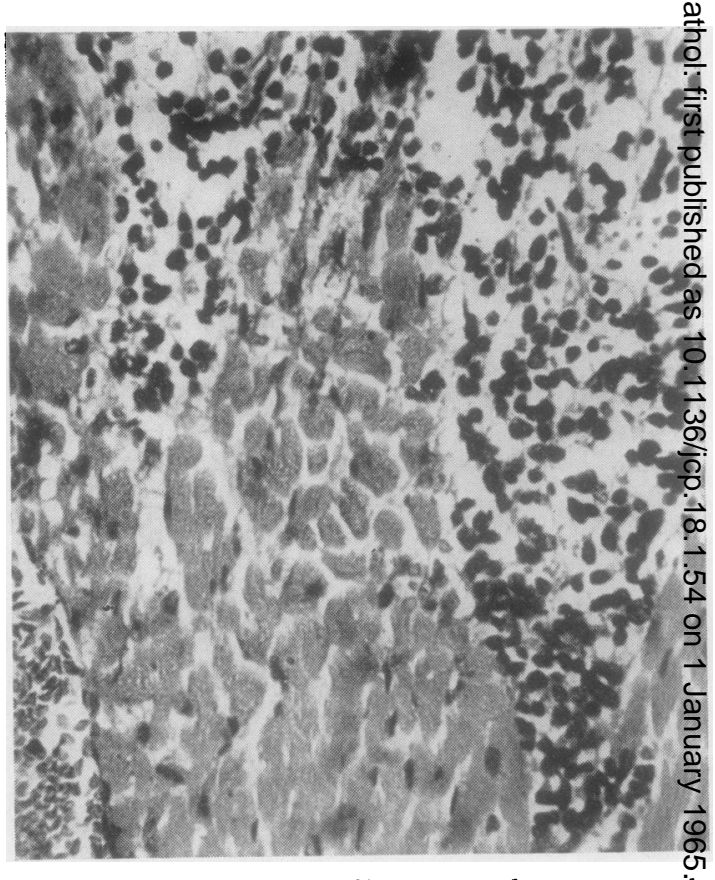

FIG. 9. Neuroblastoma infiltrating voluntary muscite (case 3). Haematoxylin and eosin $\times 320$.

\section{HISTOLOGY}

The tumour was a neuroblastoma and showed $\vec{a}$ histological pattern essentially similar to thă described in the previous two cases. No areas $\overline{\alpha f}$ haemorrhage, necrosis, or calcification were seep. Infiltration of neighbouring muscle by tumour wais evident (Fig. 9).

CASE 4

The patient was a male child and was born at home. Because of considerable difficulty in resuscitation at biren, he was admitted to St. Mary's Hospital, Manchestes, and discharged apparently well four days later. After further two days the child was readmitted with a shast history of drowsiness, reluctance to feed, and vomitiog. He was found to have meningitis, thought to be due Esch. coli. This failed to respond to antibiotic therapy (streptomycin and chloramphenicol) and the child didd 24 hours later.

\section{NECROPSY}

At necropsy the presence of meningitis was comfirmed and a right lower lobe pneumonia wa demonstrated.

A soft white tumour nodule measuring $1 \times 3 \mathrm{~cm}$. was found lying in the mid-line posteriorly j迎t 


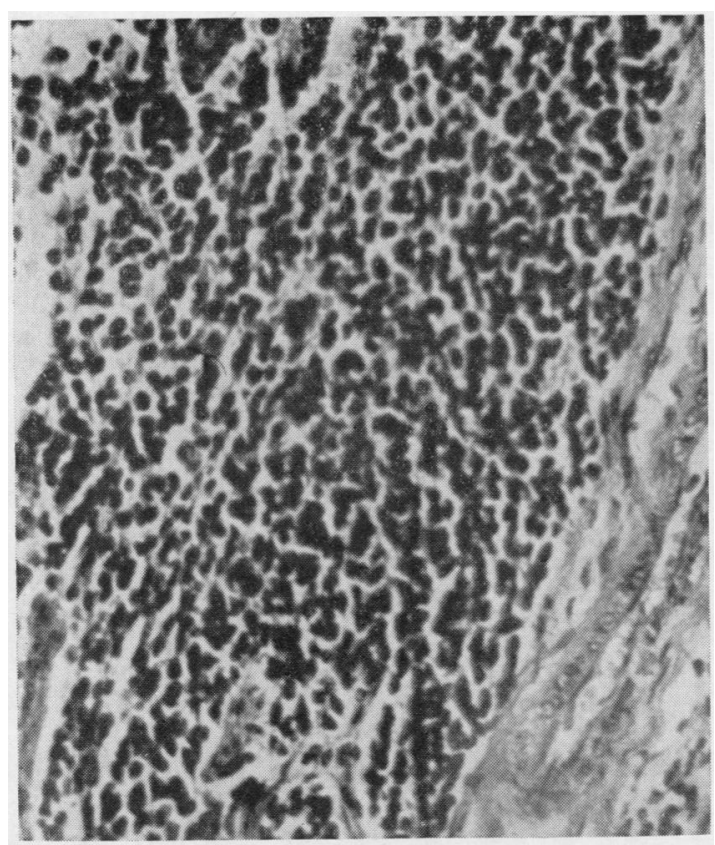

FIG. 10. Neuroblastoma (case 4) showing the more densely cellular and less differentiated pattern of this tumour. Haematoxylin and eosin $\times 320$.

below the level of the kidneys. The cut surface was creamy, white, and soft.

The remaining organs showed no significant abnormality.

\section{HISTOLOGY}

The tumour was a neuroblastoma. It showed the characteristic lobular architecture seen in the previous cases but the tumour cells themselves showed a rather greater degree of uniformity having darkly and evenly staining nuclei and very little cytoplasm. (Fig 10). In addition there was relatively little fibrillary material, as a result of which the tumour cells appeared more closely packed than in the previously described tumours. There were a few small areas of necrosis. Rosettes were not seen and the overall picture was that of a neuroblastoma showing a lesser degree of differentiation than was present in the previous cases.

\section{CASE 5}

The patient was a male child, born at home and had a birth weight of $7 \mathrm{lb}$. Delivery was normal but the mother's pregnancy was complicated by rubella in the third month. The child was said to be always rather reluctant with its feeds and gained weight only slowly.
On routine examination at a child welfare clinic his respirations were noticed to be slightly laboured but it was never considered necessary for him to be investigated in hospital. At the age of 3 months he developed an attack of apparent abdominal pain which lasted several hours and then passed off. The child died suddenly on the next day.

\section{NECROPSY}

The body was that of a well-nourished, welldeveloped male infant.

The heart was considerably enlarged with massive hypertrophy of the left ventricle. There was a coarction of the aorta of infantile type.

The lungs were congested and oedematous.

In the medulla of the right adrenal was a welldemarcated, yellowish tumour nodule $1 \mathrm{~cm}$. in diameter.

The liver was moderately enlarged and contained a number of greyish blue miliary foci up to $2 \mathrm{~mm}$. in diameter. These were visible through the capsule, and on examination of the cut surface were seen to be present throughout the organ.

The peritoneal sac contained a slight excess of clear fluid.

The brain was not examined and the remaining organs showed no abnormality apart from venous congestion.

\section{HISTOLOGY}

RIGHT ADRENAL The tumour was a neuroblastoma showing the characteristic lobular pattern and partial differentiation as described in the first three cases. A prominent feature was the presence in the more central parts of the tumour of extensive and widespread necrosis of tumour cells, whole groups of lobules appearing as necrotic 'ghosts' (Fig. 11). Foci of calcification were present in the necrotic areas. Surviving tumour was distributed mainly around the more peripheral parts of the tumour, but occasional islands and strands of surviving tumour cells were seen also lying adjacent to the stromal septa between otherwise necrotic lobules in the central zone. The tumour had clearly infiltrated the cortex.

LEFT ADRENAL There was evidence of tumour in the medulla but without cortical infiltration.

LIVER The liver contained scattered miliary foci of metastatic tumour. Strands and clumps of tumour cells were seen within the vessels of the portal tracts and growing in the sinusoids and between cords of liver cells causing destruction of these cells. 


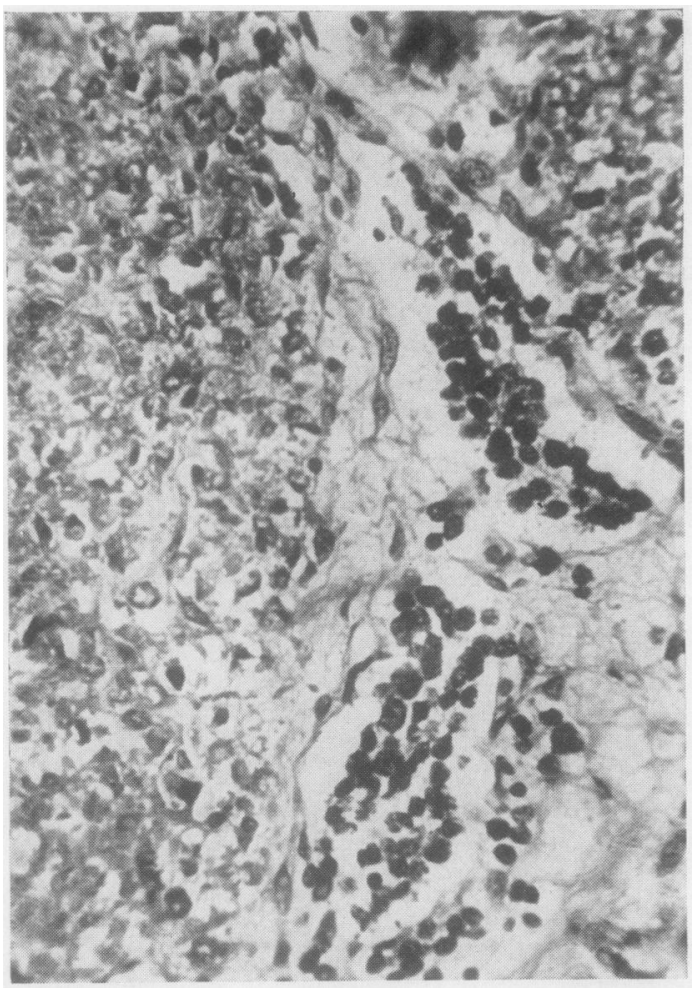

FIG. 11. Surviving tumour cells in septal tissue at the periphery of a necrotic lobule. Haematoxylin and eosin $\times$ 320 (case 5).

Between such foci of infiltration the liver appeared normal and the degree of infiltration was much less than that observed in case 1 .

\section{DISCUSSION}

The ganglia of the sympathetic nervous system and the medulla of the adrenal gland are both considered to be derived from the ectoderm of the embryonic neural crest, and neuroblastoma is a malignant embryonal tumour of these tissues. It occurs generally (though not invariably) in young children, arising either in the adrenal medulla or from some part of the abdominal, pelvic, thoracic, or cervical chains of sympathetic ganglia.

Blacklock (1934) classified the malignant neurogenic tumours of the sympathetic system as follows: A Undifferentiated sympathicoblastoma composed only of sympathogonia; B differentiated sympathicoblastoma, composed (1) of sympathogonia and sympathoblasts and (2) of ganglion cells as well as sympathogonia and sympathoblasts.

Group B2 tumours are more usually referred to as ganglioneuroblastoma, and the remainder simply as neuroblastoma. The histological pattern of the five tumours described in this report and seen most characteristically in cases $1,2,3$, and 5 may described as 'neuroblastoma with partial differentiation', and corresponds with the description of the tumours in group B1 of the above classification This type of microscopical picture is a common oge in neuroblastoma. The completely undifferentiate type of neuroblastoma, which histologically closefy resembles lymphosarcoma, accounted for only ore of the 18 tumours described in Blacklock's review $\overrightarrow{-}$

In demonstrating the minute structure, ar especially the neurofibrillary element of neuroblastoma, the use of various special stains is sometimes described (Blacklock, 1934; Evans, 1958\%, Stowens, 1957). Masson's trichrome stain and Heidenhain's iron haematoxylin and van Giesen were used on tumours in this series but, apart from tinctorial differences, revealed very little more detail of diagnostic value than that gained from the routine haematoxylin-and-eosin-stained sections.

In the case of neuroblastoma, as with other malignant tumours, amenability to treatment (by surgery and/or radiotherapy) and ultimate prognosis are paramount practical considerations. Instances of such tumours, which have differentiated towabd less malignant forms, have appeared in the literatuse since the original description of such a phenomenon by Cushing and Wolbach (1927). While a neuroblastoma which is present at birth seems in no w®y intrinsically different from one which becombs apparent later in life, and is usually recorded because congenital malignant tumours are rarities, there a登 certain reported instances which suggest that the age at which these tumours become apparent does have some bearing on ultimate prognosis.

Hepler (1943) described a case of presack neuroblastoma causing urinary obstruction in an infant of 3 weeks. After partial resection and radio therapy the child was alive and well four years later. Haber and Bennington (1963) described a case of metastasizing neuroblastoma in a 2-day- $d d$ infant. Radiotherapy and cyclophosphamide thera y were used and the child survived until the age of $\mathrm{H}_{\mathrm{g}}$ months when death occurred as a result of operative complications during attempted resection of Sिcurrent retroperitoneal tumour. Beck and Howafrd (1951) described a case of adrenal neuroblastora in a child aged 3 weeks. Following treatment child was reported alive and well six years later. \$ll three tumours can be reasonably described :as congenital. Beck and Howard justified an extensi review of the literature on neuroblastoma up to $19^{\frac{1}{1}} 1$ because of the change in prognosis associated wh that tumour. Tracing the increasing duration ${ }_{0} \mathrm{f}$ reported life spans in cases of treated neuroblaston 
they stressed that early diagnosis and treatment had become of considerably increased value, and pointed out that of a total of $\mathbf{4 7 5}$ cases of neuroblastoma reported up to that time, 47 (about 10\%) had been recorded as cures. These figures reflected a far more favourable outlook than that given by Blacklock in 1934, when, from his studies of neuroblastoma in patients at the Royal Hospital for Sick Children at Glasgow, he concluded that the average duration of life was eight or nine weeks from the first symptoms.

Stowens (1957), in a review of neuroblastoma and related tumours, drew attention to the relationship between prognosis and the age at which the tumour became apparent. Drawing his material from the files of the Armed Forces Institute of Pathology, he included in his review a series of 105 cases of neuroblastoma for which complete information was available, and, defining a survivor as one who lived at least 18 months without recurrence or metastases, found that $14(13 \%)$ of these cases were survivors. Forty-five of the 105 cases were in the first year of life when diagnosed and of these $13(29 \%)$ were survivors. The remaining survivor was one of 27 cases which fell into the age group 2 to 4 years at time of diagnosis, giving a survival rate of $4 \%$ for this age group. There were no survivors in the remaining 33 patients whose ages at diagnosis ranged from 5 years upwards and included one patient aged over 50 years. Basing his conclusions on the differences in biological behaviour of these tumours, including the disproportionately high survival rate in cases where the tumour became manifest in the first year of life, Stowens suggested that tumours, which on histological grounds are classified as neuroblastoma, actually comprise two groups. 1 A group of tumours of congenital origin resulting from disruption of normal embryogenesis of the sympathetic nervous system, which he called 'congenital neuroblastoma' and considered them always to become manifest in the first few years of life, and that about $30 \%$ of them were amenable to surgical cure and might even undergo spontaneous regression. 2 A group which resulted from malignant degeneration of fully mature elements of the sympathetic nervous system. These, he suggested, be designated 'neuroblastic sarcoma'. While anatomically indistinguishable from "congenital neuroblastoma', 'neurogenic sarcoma' was considered to occur with random frequency at all ages and to be invariably fatal.

Such a hypothesis is somewhat at variance with the more usually accepted concept of neuroblastoma, as for example as defined by Evans (1956), who described neuroblastoma as a malignant tumour of embryonic tissue which is composed of sympatho- gonia and sympathoblasts, and the continued growth of which is dependent on the proliferation of these cells. While agreeing that some neuroblastomata are congenital in origin, Stowens (1957) considered that such a postulate failed to explain the origin of this tumour when occurring in the older age groups. In such cases it would be necessary to assume that the tumours are capable of either lying dormant for considerable periods of time or having vastly different growth rates. Neither assumption seemed to him probable.

However this may be, it seems possible that the disproportionately high survival rate noticed in cases of neuroblastoma diagnosed and treated in the first year of life is not necessarily due to any intrinsic difference in tumours which happen to be present at or shortly after birth but to the fact that routine physical examinations, for one reason or another, are relatively frequent just at this age. If one such examination should reveal the presence of a palpable abdominal mass in a child possibly otherwise asymptomatic, it could lead to the rapid diagnosis and treatment of a neuroblastoma arising in the adrenal or some part of the abdominal sympathetic chain, both of which are common sites of origin for this tumour. Further, such a more or less chance finding, when it occurs before actual symptoms referable to the tumour or its metastases become manifest, may initiate treatment at a stage when its chances of success are relatively high. In this connexion it is interesting to note that in eight of Stowen's survivors, diagnosis and early surgery followed the more or less accidental finding of a palpable abdominal mass on routine physical examination in otherwise asymptomatic infants (Stowens, 1957). Again, in two of the cases already mentioned (Beck and Howard, 1951; Haber and Bennington, 1963) early treatment of neuroblastoma followed similar chance discoveries of palpable abdominal masses on clinical examination, and somewhat similar patterns of diagnosis followed by successful treatment are seen in cases described by Wyatt and Farber (1941) and by Koop, Kiesewetter, and Horn (1955). Schaffer (1960), commenting on the increasingly favourable outlook in neuroblastoma, and quoting from the reviews of Farber (1940), of Wittenborg (1950), and of Koop et al. (1955), concluded that there is general agreement that the younger the child is when the disease becomes manifest the better the prognosis. Whatever the reason, be it an intrinsic property of the congenital tumour, a tendency towards maturation to less malignant forms with the possibility of spontaneous regression, or merely that an abdominal mass is more likely to be promptly discovered in an infant or neonate than in an older child, it is apparent that 
neuroblastoma, present at birth, has a surprisingly favourable prognosis. In at least four of the five cases described in this report death was not directly due to a malignant tumour.

Since neuroblastoma, becoming manifest in the first year of life and particularly in the neonatal period, commonly presents as an abdominal mass which may be either the primary tumour or frequently a liver enlarged by metastases, the problem in diagnosis lies in distinguishing it from other causes of palpable abdominal tumour. Conditions which require consideration in the differential diagnosis include: 1 Renal enlargements not due to tumour, such as hydronephrosis and cystic kidney; 2 the enlarged livers of haemolytic disease and congenital syphilis, and the congested liver of cardiac failure seen in some cases of congenital heart lesion. The additional clinical features, serological tests for syphilis, and demonstration of rhesus or other incompatibility, are obvious aids in distinguishing these conditions. It may be mentioned that the occasional occurrence of jaundice and, more frequently, a moderate degree of anaemia in congenital neuroblastoma may cause initial confusion, especially when it is remembered that the peripheral blood film of a newborn may show considerable polychromasia together with numbers of normoblasts which may be misinterpreted as evidence of haemolytic disease when seen in association with the above-mentioned features. 3 Other abdominal tumours of infancy, including Wilms' tumour, lymphosarcoma, and the leukaemias, may be congenital, for example, five congenital malignant renal tumours recorded by Wells (1940), but the fact of the tumour being present at or shortly after birth is more in favour of its being a neuroblastoma. Blood and marrow examination will help to differentiate the leukaemias. Wilms' tumour is probably the commonest of these three tumours to present as an abdominal mass shortly after birth and is usually unilaterally situated. Intravenous pyelography may aid diagnosis by revealing pelvic and calyceal distortion by intrarenal tumour or downward displacement of the kidney by adrenal neuroblastoma. Examination of urinary catecholamines may demonstrate the alteration in cathecholamine metabolism which has been found in association with neuroblastoma but not in tumours which are not of neural crest origin (Voorhess and Gardner, 1962). Also it seems that such estimations of urinary catecholamine, its precursors and metabolites, may be of value in following up cases of treated neuroblastoma and determining the effects of therapy.

In most cases confirmation of diagnosis will follow surgical exploration when the site of the tumour will become evident. Neuroblastoma common arises from one or other adrenal, or from sympathe ganglionic tissue in the vicinity of the kidneys, although, of course, it may arise from such gang聖onic tissue at practically any site. Ultimate micro scopic diagnosis from examination of the removed or biopsied tumour will present little difficulify provided it is possible to demonstrate such charajsteristic features as cellular variation with some differentiation, presence of neurofibrillary material and perhaps rosette formation. If, however, the tumour is of the undifferentiated variety, mo:e difficulty in microscopic assessment is to be expected. Such undifferentiated forms are composed entirety of sympathogonia (Blacklock, 1934), which are small round cells with darkly staining nuclei fairly uniform size and, in the absence of much or any fibrillary material, would present a fairly uniform picture of closely packed cells very much resembling that seen in lymphosarcoma. In describing such a tumour, Blacklock drew attention to the tendency for cells at the growing margin to becone clumped into ball-like clusters similar to those found in developing sympathetic nervous tissue, and considered this a valuable feature in differentiating such a tumour from lymphosarcoma. Probably mary neuroblastomata of this type have been described sarcoma, lymphosarcoma, or round cell tumour of the adrenal, especially in reports antedating the de cription of the distinguishing characteristics of neure blastoma by Wright (1910).

I wish to thank Dr. J. K. Steward of the Children Tumour Registry, University of Manchester, for provi ing material for histological examination and for access to records for three cases; Drs. H. B. Marsden and $\bar{G}$. J. Crawford for permission to use their post-morte reports on two cases; Drs. R. M. Forrester and G. M: Komrower for allowing me to publish cases sometime their care; Dr. J. Schrager for helpful advice; and Mr. 争. Molyneux for the photographs.

\section{REFERENCES}

Beck, S. M. Jr., and Howard, P. J. (1951). Amer. J. Dis. Child., 325.

Blacklock, J. W. S. (1934). J. Path. Bact., 39, 27.

Cushing, H., and Wolbach, S. B. (1927). Amer. J. Path., 3, 203.

Evans, R. W. (1956). Histological Appearances of Tumours. Living stone, Edinburgh and London.

Farber, S. (1940). Amer. J. Dis. Child., 60, 749.

Haber, S. L., and Bennington, J. L. (1963). Arch. Path., 76, 121.

Hepler, A. B. (1943). J. Urol., 49, 777.

Koop, C. E., Kiesewetter, W. B., and Horn, R. C. (1955). Pediatri 16,652 .

Larimer, R. C. (1949). J. Pediat., 34, 365.

Schaffer, A. J. (1960). Diseases of the Newborn. Saunders, Philadelpht? and London.

Stowens, D. (1957). Arch. Path., 63, 451.

Voorhess, M. L., and Gardner, L. I. (1962). J. clin. Endocr., 22, 120,

Wells, H. G. (1940). Arch. Path., 30, 535.

Wittenborg, M. H. (1950). Radiology, 54, 679.

Wright, J. H. (1910). J. exp. Med., 12, 556.

Wyatt, G. M., and Farber, S. (1941). Amer. J. Roentgenol., 46, 485 